\title{
Isochronal Annealing Behavior of Magnesium Alloy AZ31 after Hot Deformation*
}

\author{
Xuyue Yang, Hiromi Miura and Taku Sakai \\ Department of Mechanical Engineering and Intelligent Systems, UEC Tokyo (The University of Electro-Communications), \\ Tokyo 182-8585, Japan
}

Annealing behaviors of hot-deformed magnesium alloy AZ31 were studied at temperatures of 373 to $673 \mathrm{~K}$ by optical and SEM/EBSD metallographic observation. The hot-deformed alloy had fine-grained structures developed by grain fragmentation due to continuous dynamic recrystallization (cDRX). Temperature dependence of the average grain size (D) is categorized into three temperature regions, i.e. (a) an incubation period for grain growth at $<450 \mathrm{~K}$, (b) rapid grain coarsening at $473-523 \mathrm{~K}$, and (c) normal grain growth at $T>550 \mathrm{~K}$. The number of fine grains per unit area, however, is reduced remarkably even in region (a). These lead to grain coarsening taking place continuously in the whole temperature regions. In contrast, the deformation texture scarcely changes even after full annealing at high temperatures. It is concluded that the annealing processes operating in hot-deformed magnesium alloy with cDRXed grain structures can be mainly controlled by grain coarsening accompanied with no texture change, that is continuous static recrystallization (cSRX).

(Received August 11, 2005; Accepted October 3, 2005; Published December 15, 2005)

Keywords: hot-deformed magnesium alloy, annealing, continuous dynamic and static recrystallization, grain growth, texture

\section{Introduction}

Magnesium $(\mathrm{Mg})$ is one of the lightest metals in practical use and is of growing interest as a next-generation lightweight material because of its high specific strength and excellent technological properties. ${ }^{1)}$ Most Mg alloy products have been produced by casting route, while recently request for the wrought products has been increased with development of forming process technology. $\mathrm{Mg}$ alloys show generally low cold-workability because of a few slip systems in the hexagonal close-packed (HCP) structure. One of possible methods breaking through the low ductility is to develop fine-grained structures in Mg alloys. Grain refinement could improve not only the plastic workability, but also the strength at ambient temperature. ${ }^{2)}$ Recently, severe plastic deformation (SPD) by using equal channel angular extrusion (ECAE), ${ }^{3,4)}$ multi-directional forging (MDF), ${ }^{5)}$ etc., has been carried out on the $\mathrm{Mg}$ alloys to develop a finegrained structure. The authors have studied thermo-mechanical processes for development of ultra-fine grained $\mathrm{Mg}$ alloys. They developed fine-grained structures with the grain size of submicrons by using MDF under decreasing temperature conditions and succeeded much improvement of the plastic workability and also the mechanical properties due to grain refinement. ${ }^{5}$ )

The texture control will also be important for increase in the ductility of deformed $\mathrm{Mg}$ alloys, ${ }^{6)}$ because, for example, the basal plane becomes perpendicular to the forging direction in high strain during hot deformation. ${ }^{7)}$ Further improvement of the ductility of deformed $\mathrm{Mg}$ alloys will be carried through the texture control due to annealing treatment following plastic deformation. There have been a few reports on the annealing behaviors of hot-deformed $\mathrm{Mg}$ alloys. The annealing characteristics and the texture changes in a widely used Mg alloy, e.g. AZ31, have been almost unknown, as far

*This Paper was Originally Published in Japanese in J. Japan Inst. Metals 68 (2004) 946-951. as the authors know. ${ }^{8)}$

The present work aimed to investigate the annealing characteristic of a Mg alloy AZ31 after hot deformation. The structural changes taking place during hot deformation and subsequent isochronal annealing were systematically studied at various reheating temperatures. Changes in the grain structure and the texture developed during and after hot deformation are analyzed in detail, and the mechanisms of annealing processes also are discussed.

\section{Experimental Procedure}

A commercially produced Mg alloy AZ31 was provided as a hot-extruded rod with a diameter of $19 \mathrm{~mm}$. The chemical composition was as follows; $\mathrm{Al} 2.68, \mathrm{Zn} \mathrm{0.75,} \mathrm{Mn} \mathrm{0.68,} \mathrm{Cu}$ 0.001 , Si $0.003, \mathrm{Fe} 0.003$, and balance $\mathrm{Mg}$ (all in mass\%). Cylindrical samples of $8 \mathrm{~mm}$ in diameter and $12 \mathrm{~mm}$ in height were machined from the rod parallel to the extrusion direction. Then 6 evenly spaced annular grooves of 0.12 $\mathrm{mm}$ depth were machined into the end faces of cylinders in order to promote the retention of a graphite lubricant (DAG 154). The samples were annealed at $733 \mathrm{~K}$ for $7.2 \mathrm{ks}$ and then furnace cooled, leading to the evolution of equiaxed grains with an average size of about $22 \mu \mathrm{m}$.

Compression tests were carried out at constant true strain rates on a testing machine equipped with a water quenching apparatus, ${ }^{7}$ ) which made it possible to quench the samples within $1.5 \mathrm{~s}$ after deformation was ceased. The samples were deformed up to strains of 1.2 at $573 \mathrm{~K}$ and at two true strain rates of $3 \times 10^{-3}$ and $3 \times 10^{-1} \mathrm{~s}^{-1}$, followed by quenching in water. They were cut to plates with 2 to $3 \mathrm{~mm}$ thickness parallel to the compression axis, and then annealed for $1 \mathrm{ks}$ in air using a muffle furnace at temperatures ranging from 373 to $673 \mathrm{~K}$. Each plate was mechanically and electrolytically polished and then etched in a solution of $6 \%$ picric acid and 94\% methanol. As-deformed and the subsequent annealed microstructures were examined by using optical microscopy (OM) and orientation imaging microscopy (OIM). The 


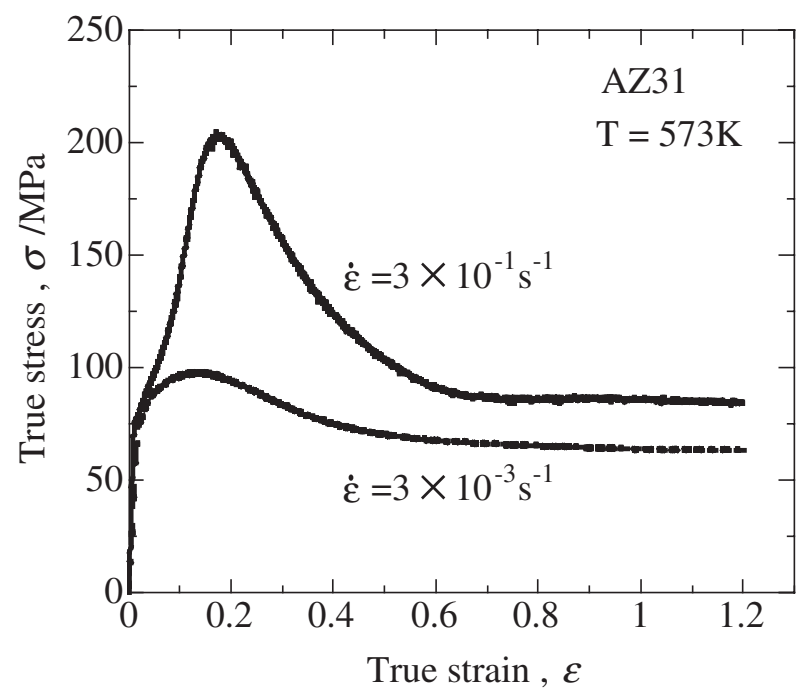

Fig. 1 Effect of strain rate on true stress-true strain curves for AZ31 alloy at $573 \mathrm{~K}$.

distributions of grain size were analyzed by using an image analysis program of "AnalySIS" (Soft Imaging System Co.). Crystallographical orientation and texture changes were examined by SEM/OIM. The Vickers hardness was measured on each plate at room temperature under a condition of $3 \mathrm{~N}$.

\section{Results and Discussion}

\subsection{Hot deformation behaviors}

Figure 1 shows typical true stress-true strain curves for the AZ31 alloy compressed at $573 \mathrm{~K}$ and at strain rates of $3 \times$ $10^{-3}$ and $3 \times 10^{-1} \mathrm{~s}^{-1}$. It is seen in Fig. 1 that the flow stresses as well as the shape of the flow curves are sensitively affected by strain rate. The flow curve at $3 \times 10^{-3} \mathrm{~s}^{-1}$ shows a smooth stress peak following gradual strain hardening in relatively low strain, followed by strain softening and steadstate flow in high strain. The flow shape at a high strain rate of $3 \times 10^{-1} \mathrm{~s}^{-1}$, in contrast, indicates a sharper stress peak following rapid strain hardening and then rapid strain softening and steady-state flow in high strain. These suggest that mechanisms of structural change operating during deformation depend sensitively on strain rate.

\subsection{Structural changes during hot deformation}

It is well known that new grain structures are developed in relatively low strain during warm and hot deformation of $\mathrm{Mg}$ alloys. ${ }^{2,7)}$ Changes in the volume fraction and the average size of new grains ( $V_{\text {rex }}$ and $D_{\text {rex }}$ ) with deformation at $573 \mathrm{~K}$ are shown in Fig. 2. New grains start to develop at around the stress peak and $V_{\text {rex }}$ rises rapidly during work softening and then approaches a saturation value in high strain. In contrast, $D_{\text {rex }}$ in the new grained regions decreases slightly during work softening and approaches a saturation value. It is considered that $D_{\text {rex }}$ developed at low strains is roughly similar to that in high strain. ${ }^{7)}$ With increasing strain rate, the strain at which $V_{\text {rex }}$ approaches a constant increases and conversely $D_{\text {rex }}$ in high strain decreases from 4 to $2.7 \mu \mathrm{m}$.

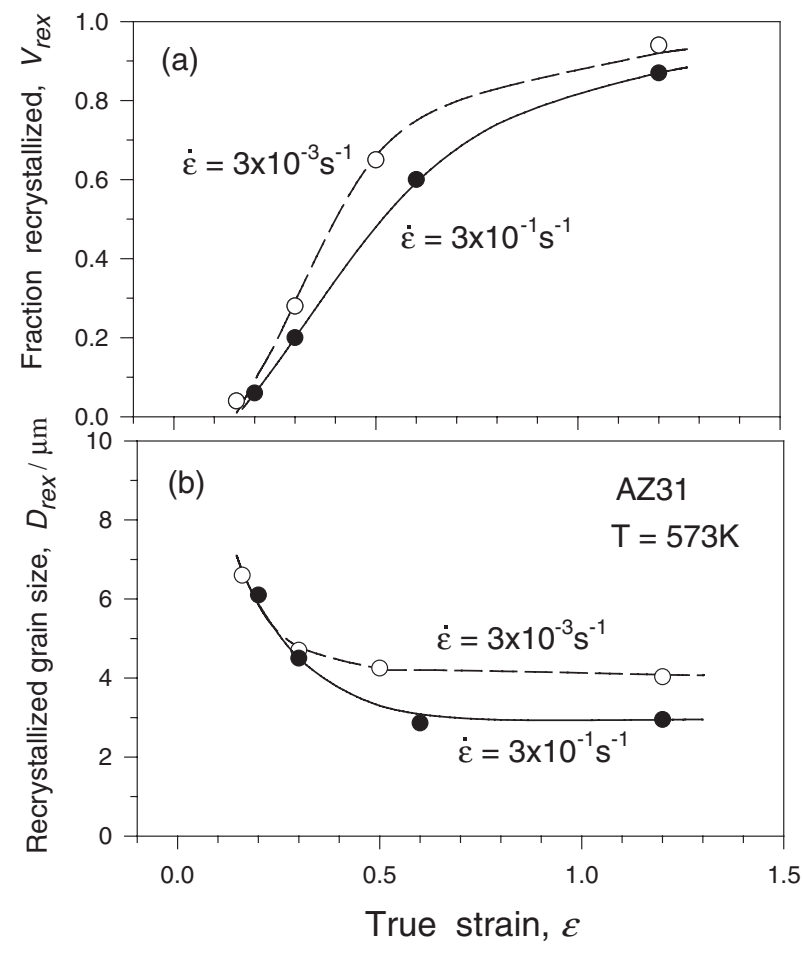

Fig. 2 Effects of strain and strain rate on (a) the volume fraction $\left(V_{\text {rex }}\right)$ and (b) the average size of new grains $\left(D_{\text {rex }}\right)$ for AZ31 alloy deformed at $573 \mathrm{~K}$.

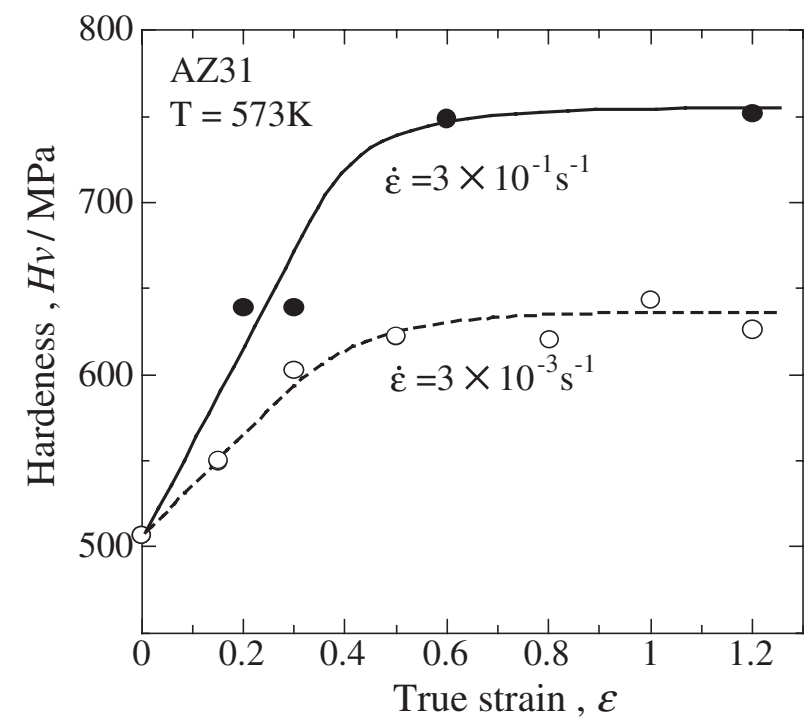

Fig. 3 Effect of strain rate on changes in room-temperature hardness with deformation at $573 \mathrm{~K}$ for AZ31 alloy.

Figure 3 shows strain dependence of the room temperature hardness $\left(H_{\mathrm{v}}\right)$ for the hot-deformed $\mathrm{Mg}$ alloy. $H_{\mathrm{v}}$ increases monotonously with strain and approaches to a saturation in high strain irrespective of strain hardening and softening in the flow curves (Fig. 1). The increasing rate of $H_{\mathrm{v}}$ and the saturation value in high strain are higher with increasing strain rate. The $H_{\mathrm{v}}-\varepsilon$ relationships in Fig. 3 may correspond to those between the reciprocal $D_{\text {rex }}$ and strain in Fig. 2 . Namely, $H_{\mathrm{v}}$ increases with reduction of the average grain size 

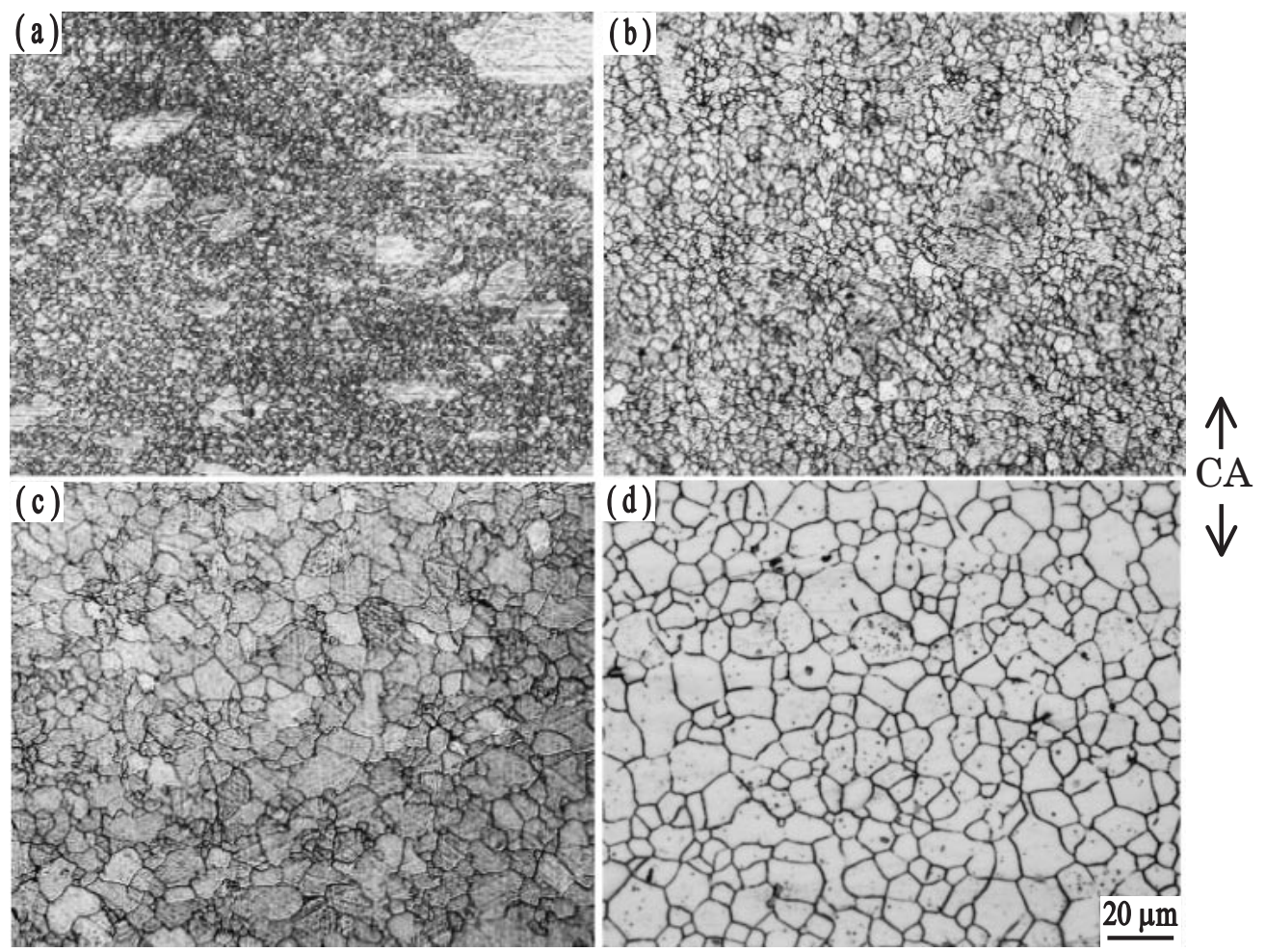

Fig. 4 Microstructures developed in AZ31 alloy (a) deformed to $\varepsilon=1.2$ at $573 \mathrm{~K}$ with a strain rate of $3 \times 10^{-1} \mathrm{~s}^{-1}$, followed by annealing for $10^{3} \mathrm{~s}$ at (b) $473 \mathrm{~K}$, (c) $523 \mathrm{~K}$ and (d) $673 \mathrm{~K}$. CA indicates the compression axis.

$D$ due to increasing the volume fraction of new grains, and approaches to a saturation value in high strain, where $D_{\text {rex }}$ is almost constant. This may suggest that the room temperature hardness of hot-deformed $\mathrm{Mg}$ alloy can be controlled mainly by grain refinement rather than increasing dislocation densities introduced by deformation. These results will be discussed in detail in section 3.6.

\subsection{Structural changes during isochronal annealing}

Figure 4 shows a series of typical optical microstructures evolved before and after annealing at various temperatures for the AZ31 alloy deformed to $\varepsilon=1.2$ at $573 \mathrm{~K}$ and at $3 \times 10^{-1} \mathrm{~s}^{-1}$. The authors ${ }^{7,9,10)}$ previously discussed that new grains evolved in $\mathrm{Mg}$ alloy during hot deformation can result from a kind of strain-induced continuous recrystallization, but not from conventional discontinuous recrystallization. The process of new grain formation in hot-deformed $\mathrm{Mg}$ alloy is described briefly as follows: (1) Kink bands are developed frequently in grain interiors at low strains and the misorientation and the number of the boundaries of kink bands rise rapidly with deformation because HCP structure have a limited number of slip systems. (2) Their intersections take place with deformation, leading to fragmentation of original grains. The average size of the fragmented domain is almost the same as that of new grains. (3) It is concluded, therefore, that the new grain formation can result from grain fragmentation by development of kink band and so be controlled by deformation-induced continuous reactions assisted by dynamic recovery. This is generally called as in-situ dynamic recrystallization or continuous dynamic recrystallization $(c D R X)$. It should be noted that new grains were not fully developed throughout the material even in high strain, as can be seen in Fig. 4(a) and discussed in elsewhere. ${ }^{7)}$

Figures 4(b) to (d) show that such strain induced new grains coarsen homogeneously with increase in reheating temperature. After annealing at temperatures below $473 \mathrm{~K}$, some rather coarse grains still remain and are clearly distinguishable from surrounding fine grains [Fig. 4(b)]. After annealing at $T \geq 503 \mathrm{~K}$ [Figs. 4(c) and (d)], it is difficult to distinguish the coarse grains from the surrounding ones which coarsen homogeneously, finally leading to a fully development of an almost equiaxed grain structure.

Figure 5 shows changes in the average grain size $(D)$ in the whole area with annealing temperature for the AZ31 alloy deformed to $\varepsilon=1.2$ at $573 \mathrm{~K}$ and at strain rates of $3 \times 10^{-3}$ and $3 \times 10^{-1} \mathrm{~s}^{-1}$. $D$ was measured by using a linear line intercept method. $D$ is hardly changed during annealing at low temperature $<473 \mathrm{~K}$. $D$ increases rapidly at $473-523 \mathrm{~K}$ and then increases slowly with rising temperature. Such a grain size change looks like that occurring in conventional deformed cubic metals during annealing. ${ }^{11)}$ The temperature range from 300 to $450 \mathrm{~K}$ may correspond to an incubation region for recrystallization, when a period of annealing is $1 \mathrm{ks}$, that from 473 to $523 \mathrm{~K}$ a recrystallization region and the high temperature side above around $550 \mathrm{~K}$ a region for normal grain growth, respectively. The temperature, at which rapid grain growth takes place, falls a little with increasing strain rate. This result also is similar to that for annealing of deformed cubic metals. ${ }^{11)}$ 


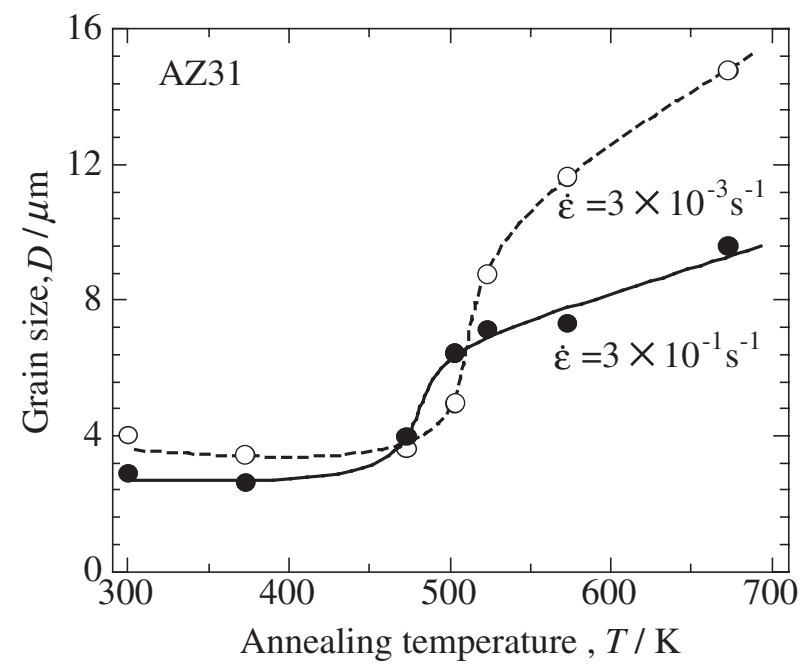

Fig. 5 Effect of strain rate on changes in average grain size in the whole area, $D$, with annealing temperature for AZ31 alloy deformed to $\varepsilon=1.2$ at $573 \mathrm{~K}$.

\subsection{Quantitative analysis of microstructures and texture}

Typical grain size distributions before and after annealing at 373 to $673 \mathrm{~K}$ are shown in Fig. 6 for the samples deformed to $\varepsilon=1.2$ at $573 \mathrm{~K}$ and at $3 \times 10^{-1} \mathrm{~s}^{-1}$. The distribution at $373 \mathrm{~K}$ is almost similar to that for as-deformed state and shows a bimodal shape with two peaks at around 2 and $20 \mu \mathrm{m}$. The fine grains correspond to those newly developed by hot deformation and the coarser ones to the remained original grains [see Fig. 4(a)]. Upon annealing at $T>473 \mathrm{~K}$, the relative frequency of fine grains rapidly decreases and the range of coarse grain sizes gradually increases with increase in temperature. With further annealing at $T>523 \mathrm{~K}$, the grain size distribution is similar to a log-normal type with a single peak at around $20 \mu \mathrm{m}$ [see Figs. 6(e) and (f)].

Figure 7 shows changes in the number of fine grains per unit area, $\mathrm{N}$, less than $10 \mu \mathrm{m}$ in diameter with annealing temperature. It is remarkable to note that $\mathrm{N}$ decreases clearly even at low temperatures of $T<450 \mathrm{~K}$. This is in contrast with the result of the average grain size in Fig. 5, i.e. there is no remarkable grain coarsening in this temperature region. It is concluded from Fig. 7 that grain growth starts to take place mainly in the fine-grained regions simultaneously just after annealing even at low temperature [Figs. 6(a) to (c)]. Further annealing in the temperature region between 473 and $523 \mathrm{~K}$ leads to a rapid grain coarsening accompanied by a rapid decrease in $\mathrm{N}$. Then the grain size distribution changes from a double peak to a single peak type, as shown in Figs. 6(c) and (d). During this process, some coarse grains containing high density dislocations [see Fig. 8(a)] may be consumed by recovered growing grains in the surroundings. During annealing at $T>523 \mathrm{~K}$, the average grain size gradually increases accompanied with a little decrease in $\mathrm{N}$, suggesting the occurrence of normal grain growth.

Typical OIM micrographs with the inverse pole figures for compression direction are shown in Fig. 8. Figures 8(a), (b) and (c) correspond to the microstructures in Figs. 4(a), (b) and (d). Disorientation $(\theta) \theta>2^{\circ}, \theta>4^{\circ}$ are delineated by thin white and black lines, while high-angle boundaries

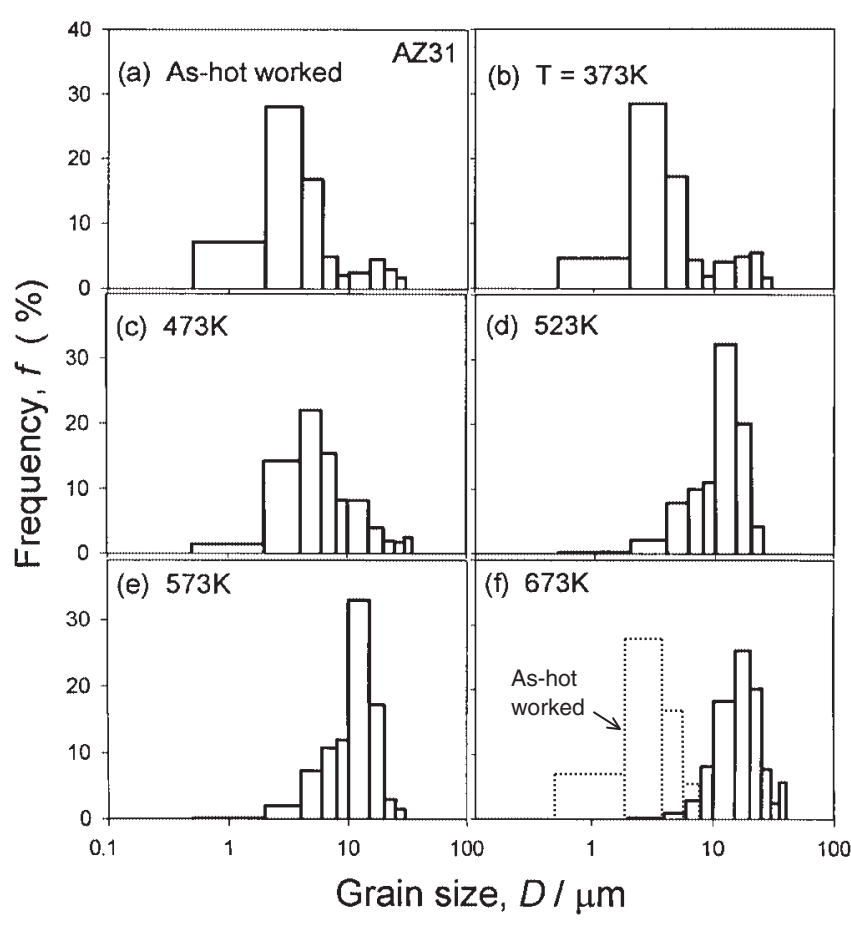

Fig. 6 Changes in grain size distribution with annealing temperatures for AZ31 alloy deformed to $\varepsilon=1.2$ at $3 \times 10^{-1} \mathrm{~s}^{-1}$ and at $573 \mathrm{~K}$

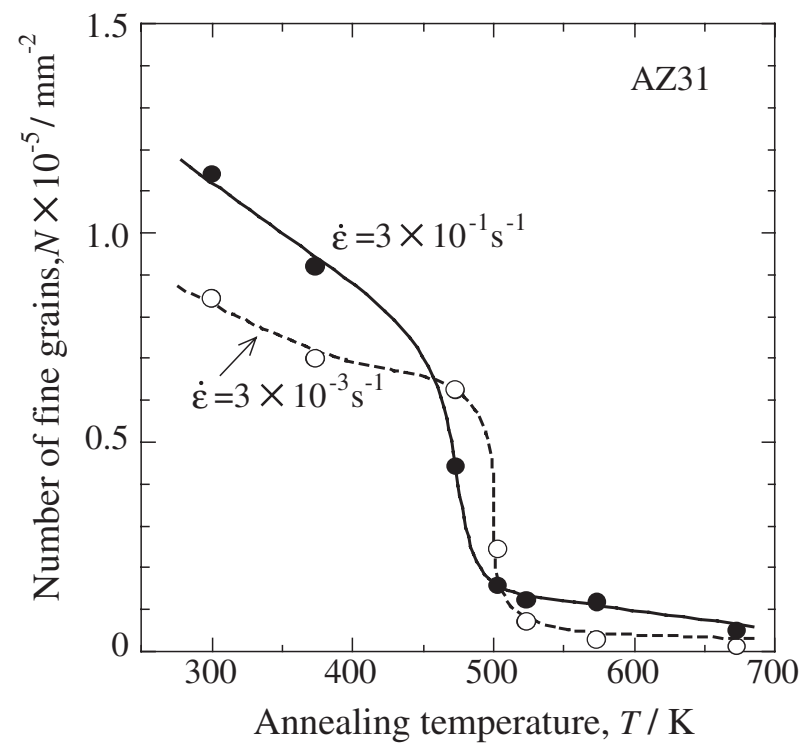

Fig. 7 Effect of strain rate on the relationship between the number of fine grains with less than $10 \mu \mathrm{m}$ in diameter per unit area, $\mathrm{N}$, and annealing temperature for AZ31 alloy deformed to $\varepsilon=1.2$ at $573 \mathrm{~K}$.

including kink bands ${ }^{7)}$ with misorientations more than $15^{\circ}$ are by bold black lines. Different colors for each grain correspond to different crystallographic orientations. It is clearly seen in Fig. 8 that most of the grains developed have a strong texture near (0001) compared with those near (1010) or $(1 \overline{2} 0)$. It is known ${ }^{7)}$ that extruded rod of Mg alloys has a strong texture in which the basal plane of the HCP lattice lies parallel to the extrusion direction (and so the compression direction in the present case). The basal plane rotates 

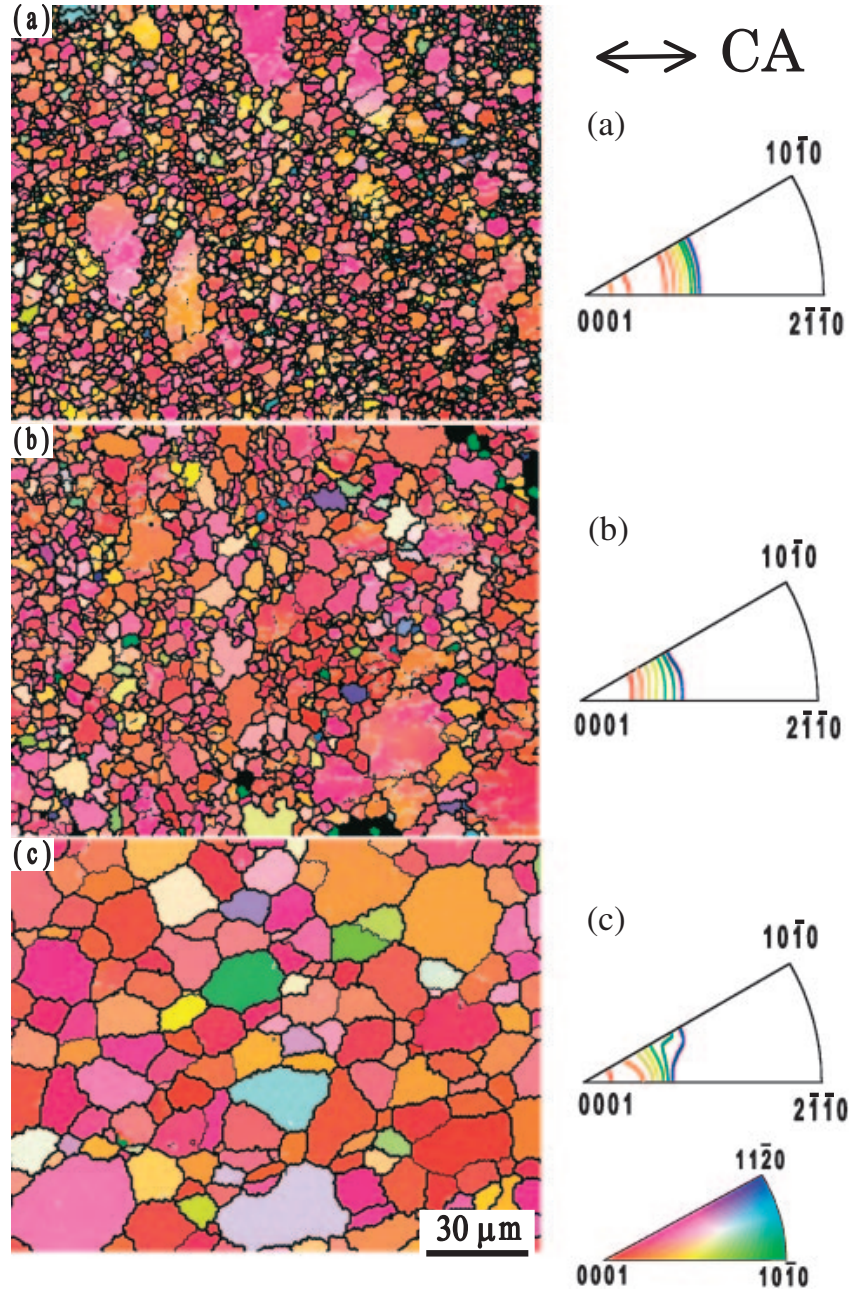

Fig. 8 OIM micrographs and inverse pole figures of AZ31 alloy (a) deformed to $\varepsilon=1.2$ at $573 \mathrm{~K}$ with at $3 \times 10^{-1} \mathrm{~s}^{-1}$, followed by annealing for $10^{3} \mathrm{~s}$ at (b) $473 \mathrm{~K}$ and (c) $673 \mathrm{~K}$. Thin white lines correspond to boundaries of misorientation $>2^{\circ}$, thin black line $>4^{\circ}$ and thick black lines $>15^{\circ}$, respectively. Colors correspond to crystallographic orientations indicated in the inverse pole figure.

gradually from around $0^{\circ}$ to near $90^{\circ}$ to the compression axis with deformation. It is remarkable to note in Fig. 8 that such a deformation texture with (0001) perpendicular to the compression axis exists stably even after full annealing at high temperatures. It is concluded that strain-induced fine grains evolved in $\mathrm{Mg}$ alloys can grow remarkably and, in contrast, the deformation texture is scarcely changed with annealing.

\subsection{Static restoration mechanisms operating in cDRXed Mg alloys}

The present experimental results described above, can be summarized as the followings.

(1) The grain size distribution shows a single peak type during annealing at $T>500 \mathrm{~K}$ where rapid grain coarsening takes place [Figs. 6(d) to (f)].

(2) Grain coarsening takes place continuously during annealing even at low temperatures (Figs. 5 and 7).

(3) The deformation texture is scarcely changed during grain coarsening (Fig. 8).

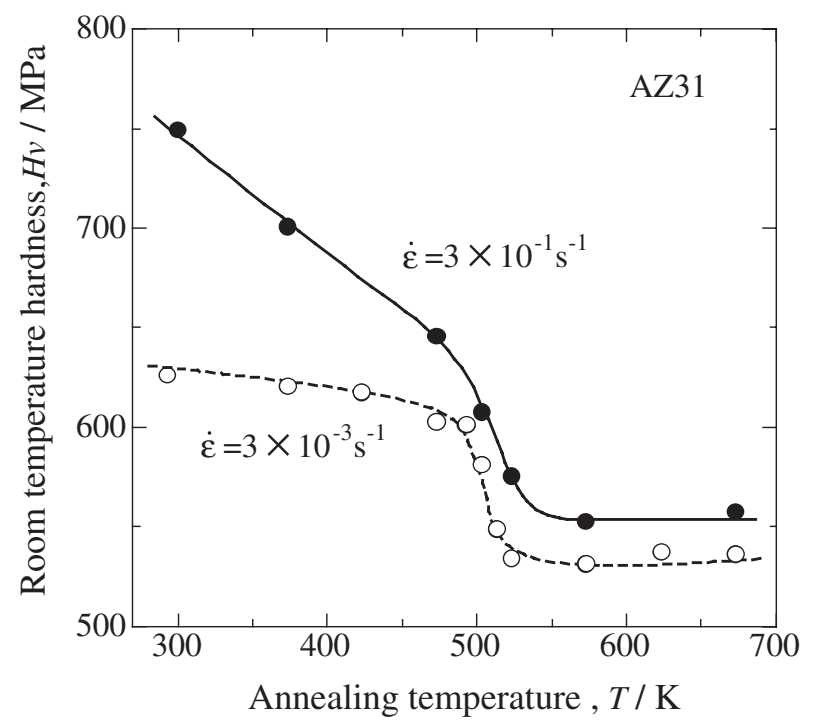

Fig. 9 Effect of strain rate on changes in room-temperature hardness with annealing temperature for AZ31 alloy deformed to $\varepsilon=1.2$ at $573 \mathrm{~K}$.

Such annealing behaviors are in contrast with those for conventional deformed cubic metals, in which discontinuous recrystallization takes place accompanied with nucleation of new grains and their long range grain boundary migration. It is concluded, therefore, that annealing processes operating in hot-deformed $\mathrm{Mg}$ alloy is controlled mainly by grain coarsening accompanied with no texture change, that is continuous static recrystallization (cSRX). It is interesting to note that dynamic evolution of new grains in $\mathrm{Mg}$ alloys results from continuous DRX taking place during hot deformation, as described above, and continuous static recrystallization can subsequently operate in cDRXed grain structure during annealing.

It is suggested by Fig. 8 that the texture of wrought $\mathrm{Mg}$ alloy may be hardly controlled by using conventional methods, i.e. plastic working followed by annealing. It is also seen in Fig. 8(a) that dislocation boundaries with low angle misorientations are developed in remained coarse grains, and the cumulative misorientations at near the original grain boundaries are within a few degrees, i.e. these is almost no lattice orientation gradient evolved. ${ }^{7)}$ This is in contrast with the cases of hot-deformed grains and conventional DRXed grains in cubic metals. Namely, some high orientation and strain gradients are always formed near the grain boundaries, leading to nucleation with various orientations followed by long range grain boundary migration. ${ }^{11-14)}$ Thus, deformation process accompanied with various strain paths, such as multi-directional forging (MDF), ${ }^{5)}$ may be useful for texture modification of $\mathrm{Mg}$ alloy.

\subsection{Effects of hot deformation and annealing on room- temperature hardness}

Figure 9 shows changes in room-temperature hardness $\left(H_{\mathrm{v}}\right)$ with annealing temperature for the AZ31 alloy deformed to $\varepsilon=1.2$ at $573 \mathrm{~K}$ and at strain rates of $3 \times$ $10^{-3}$ and $3 \times 10^{-1} \mathrm{~s}^{-1}$. $H_{\mathrm{v}}$ begins to decrease gradually at low temperatures, then rapidly at $473-523 \mathrm{~K}$, and finally approaches a constant value in high temperature above 


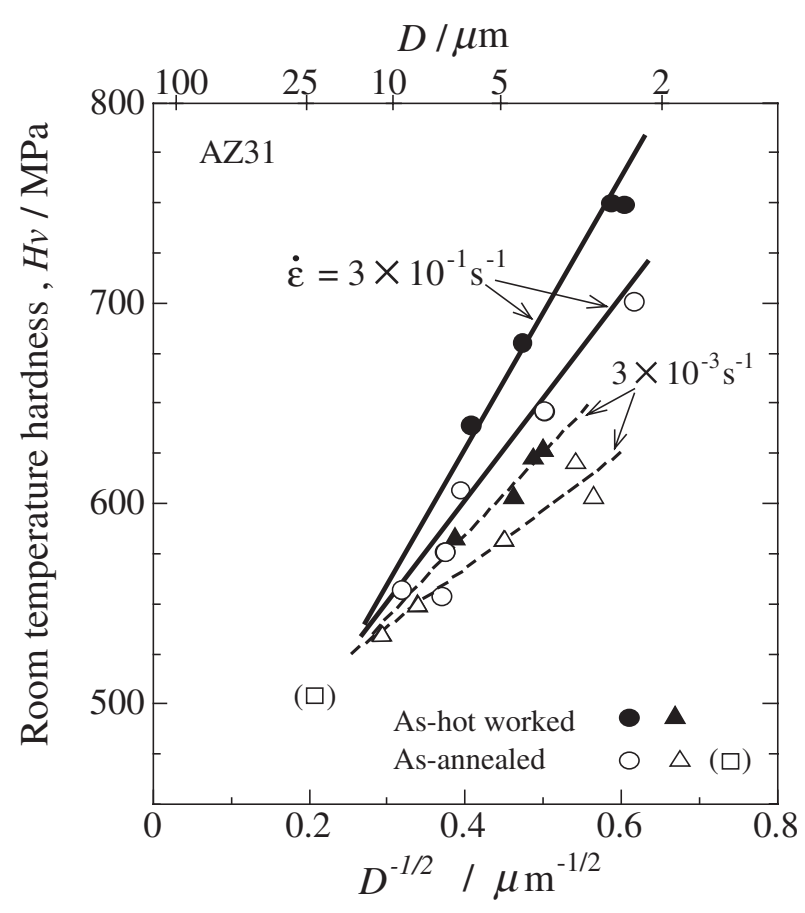

Fig. 10 Effect of strain rate on the relationships between room-temperature hardness and average grain size for AZ31 alloy (a) as-hot worked to $\varepsilon=1.2$ at $573 \mathrm{~K}$ and at various strain rates (solid marks) and (b) followed by annealing for $10^{3} \mathrm{~s}$ at various temperatures (open marks). ( $\square$ ) shows the data for a full-annealed initial sample.

$523 \mathrm{~K}$. Such $H_{\mathrm{v}}-T$ relationships are roughly similar to the $\mathrm{N}-T$ ones in Fig. 7 compared with the data of $D$ in Fig. 5. The values of $H_{\mathrm{v}}$ and $\mathrm{N}$ at $3 \times 10^{-1} \mathrm{~s}^{-1}$ are always larger than those at $3 \times 10^{-3} \mathrm{~s}^{-1}$ except the temperature range from 473 to $523 \mathrm{~K}$. Effect of strain rate on the changes in $H_{\mathrm{v}}$ at $473-523 \mathrm{~K}$ is different from those for $\mathrm{N}$ or $D$ in hot-deformed structure. This will be discussed in the following.

The room temperature hardness of hot-deformed $\mathrm{Mg}$ alloy should be influenced not only by deformation-induced dislocation substructures, but also new fine grains developed. The relationships between $H_{\mathrm{v}}$ and $D$ developed before and after full annealing are summarized in Fig. 10 according to the Hall-Petch equation, i.e. $H_{\mathrm{v}}=H_{0}+k D^{-1 / 2}$, where $H_{0}$ and $\mathrm{k}$ are experimental constants. It is seen clearly in Fig. 10 that $H_{\mathrm{v}}-D$ relationships for as-deformed and full-annealed states can be approximated by a different Hall-Petch equation. The relationship is also dependent sensitively on strain rate. It is considered that the difference in $H_{\mathrm{v}}$ for a same grain size in as-deformed and full annealing state can result from reduction in dislocation densities as well as fine grains during annealing. A Hall-Petch slope $(k)$ at a lower strain rate should be smaller compared with that at high strain rate, because dynamic recovery takes place more frequently during deformation.

It is concluded from the above examination, therefore, that a main factor controlling the value of $H_{\mathrm{v}}$ for hot-deformed and subsequent full-annealed $\mathrm{Mg}$ alloy can result from grain refinement or coarsening due to the Hall-Petch relationship, but a little from changes in dislocation densities due to dynamic or static recovery. This is in contrast with that for conventional strain-hardened cubic metals, ${ }^{11)}$ i.e. changes in $H_{\mathrm{v}}$ during hot deformation and subsequent annealing can result mainly from increase or decrease in dislocation densities in substructures.

\section{Conclusions}

Isochronal annealing behaviors of hot-deformed $\mathrm{Mg}$ alloy AZ31 were studied at temperatures from 373 to $673 \mathrm{~K}$. The main results obtained are summarized as follows.

(1) Temperature dependence of the average grain size (D) is categorized into three temperature regions, i.e. (a) an incubation period of grain growth at $<450 \mathrm{~K}$, (b) rapid grain coarsening at $473-523 \mathrm{~K}$ and (c) normal grain growth at $T>550 \mathrm{~K}$. The number of fine grains per unit area, however, is reduced remarkably even in region (a). It is concluded, therefore, that grain growth takes place continuously during annealing in the whole range from low to high temperature.

(2) A bimodal distribution of grain size in as-deformed state changes to a single peak type after rapid grain coarsening taking place in medium to high temperature. The relative frequency of fine grains decreases rapidly and that of coarse grains increases gradually with annealing.

(3) The deformation texture with the basal plane (0001) perpendicular to the compression axis can exist stably at high temperature and so scarcely change even after full annealing.

(4) It is concluded from the results (1) to (3) that the annealing processes operating in hot-deformed $\mathrm{Mg}$ alloy with fine grains, developed by continuous dynamic recrystallization (cDRX), are controlled mainly by grain growth accompanied with no texture change, that is continuous static recrystallization (cSRX).

(5) The relationships between room-temperature hardness and average grain size for as-deformed and fullannealed states can be approximated by different Hall-Petch relationships, which also depend sensitively on strain rate during hot deformation.

(6) The main reason for the changes in the room temperature hardness during hot deformation and subsequent annealing can result from grain refinement or coarsening, and a little from change in the dislocation density due to dynamic or static recovery.

\section{Acknowledgments}

The authors wish to acknowledge the financial support by a Grant-in-Aid for Basic Research (C) (16560627) from the Ministry of Education, Science and Culture and the Light Metals Educational Foundation, Japan.

\section{REFERENCES}

1) B. L. Mordike and T. Ebert: Mater. Sci. Eng. A 302 (2001) 37-45.

2) H. Watanabe, T. Mukai and K. Higashi: J. Jpn. Inst. Light Met. 51 (2001) 503-508.

3) Yamashita, Z. Horita and T. G. Langdon: Mater. Sci. Eng. A 300 (2001) 142-147. 
4) Y. Yoshida, L. Cisar, S. Camado and Y. Kojima: J. Jpn. Inst. Light Met. 52 (2002) 559-565.

5) J. Xing, H. Sohda, X. Yang, H. Miura and T. Sakai: Mater. Trans. 46 (2005) 1646-1650.

6) M. Yoshinaga: Met. Phys. 10 (1964) 91-100.

7) X. Yang, H. Miura and T. Sakai: Mater. Trans. 44 (2003) 197-203.

8) A. Yamamoto, M. Kakishiro, M. Ikeda and H. Tsubakino: Mater. Sci. Forum 449-452 (2004) 669-672.

9) X. Yang, H. Miura and T. Sakai: Mater. Sci. Forum 419-422 (2003) $515-520$.
10) X. Yang, H. Miura and T. Sakai: Mater. Sci. Forum 426-432 (2003) 611-616.

11) F. J. Humphreys and M. Hatherly: Recrystallization and Related Annealing Phenomena, (Pergamon, 1995) pp. 127-392.

12) T. Sakai and J. J. Jonas: Acta Metall. 32 (1984) 189-209.

13) N. Hansen: Evolution of Deformation Microstructure in 3D, ed. by C. Gundlack et al. (Riso National Lab., Denmark, 2004) pp. 13-32.

14) A. M. Wusatowska-Sarnek, H. Miura and T. Sakai: Mater. Trans. 42 (2001) 2452-2459. 